\title{
Complex Zeros of the Modified Bessel Function $K_{n}(Z)^{*}$
}

\author{
By R. Parnes
}

\begin{abstract}
The complex zeros of $K_{n}(Z)$ are computed for integer orders $n=2(1) 10$, to 9D figures, using an iterative interpolation scheme.
\end{abstract}

1. Introduction. The investigation of wave propagation and scattering in elastic media is often performed by means of integral transform methods. The analysis of such problems in cylindrical coordinates often leads to waves whose transformed potential functions are expressed in terms of modified Bessel functions. In particular, the potentials for outgoing radiating waves which decay with increasing distance from the source are expressed in terms of modified Bessel functions of the second kind, $K_{n}(Z)$. In the course of a recent study using the Laplace transform, it was necessary to determine complex zeros of $K_{n}(Z)$ in order to locate poles of the solution required for the inversion. It is believed that the tabulated zeros given below will permit the evaluation of several significant scattering problems.

Several methods for the evaluation of zeros of Bessel functions, notably by means of asymptotic expansions, have been given by Olver [1] and Luke [2]. The methods developed by Olver, however, are not entirely applicable in the present case, since the convergence only improves with large orders of $n$. On the other hand, the rational approximations given by Luke have been proved, under appropriate restrictions of the parameters, to converge in the first quadrant; convergence for $|\arg Z|<\pi$ has recently been established by Fields [3]. It should be observed that Cochran and Hoffspiegel [4] have extended the work of Olver for determining the positive real or purely imaginary zeros of Hankel functions with respect to noninteger orders when the variable is held fixed. However, their methods are inapplicable, since the zeros of the modified Bessel functions are seen to be always complex. A method for the evaluation of complex zeros of cylinder functions was given by Döring [5] based on MacMahon and Olver expansions. Tabulated values were given for some zeros of Hankel functions $H_{n}(Z)$ which can be related to those of $K_{n}(Z)$.

The zeros presented below to $9 \mathrm{D}$ significant figures were calculated according to a method described in the following section.

2. Formulas and Method. The modified Bessel function of the second kind is given by the expression

Received January 2, 1971, revised December 13, 1971.

AMS 1970 subject classifications. Primary 30A08, 33A40; Secondary 35L05.

Key words and phrases. Bessel functions, complex zeros.

* The research reported in this paper was supported by the National Science Foundation under grant GK-14514. 


$$
\begin{aligned}
K_{n}(Z)= & \frac{1}{2} \sum_{r=0}^{n-1}(-1)^{r} \frac{(n-r-1) !}{r !}(2 / Z)^{n-2 r} \\
& +(-1)^{n+1} \sum_{r=0}^{\infty} \frac{(Z / 2)^{n+2 r}}{r !(n+r) !}\left\{\ln (Z / 2)-\frac{1}{2}[\psi(r+1)+\psi(n+r+1)]\right\},
\end{aligned}
$$

where

$$
\psi(n+r+1)=1+1 / 2+1 / 3+\cdots+1 /(n+r)-\gamma
$$

and where $\gamma=0.5772156649015329 \ldots$.

Due to the logarithmic term, a branch cut is required and is taken along the negative real axis, with the branch point at the origin, such that $-\pi<\arg Z \leqq \pi$.

Following Watson [6], it may be shown that the finite zeros for the given branch lie in the quadrants for which $\pi / 2<|\arg Z|<\pi$, i.e., all zeros have $\operatorname{Re} Z<0$ but cannot lie on the negative real axis. Furthermore, the zeros are known to occur in complex conjugate pairs.

Letting $K_{n}(Z)=R e^{i \theta}$, the local region of the zeros is first determined by tracing the argument $\theta$ along a sequence of closed contours. Since $K_{n}(Z)$ contains no poles for $Z \neq 0$, use is made of the theorem which states that, for such an analytic function, the number of zeros in a region is $1 / 2 \pi$ times the change of argument $\theta$ of the function in going around a closed contour enclosing the region [7].

Having localized the region of the zeros $Z_{0}$ of $K_{n}(Z)$ by the above procedure, a grid is established within the region with a spacing $\Delta^{(1)}$ for both the real and imaginary parts of $Z=a+i b$, giving a sequence of points $\left\{a_{k}^{(1)}, b_{k}^{(1)}\right\}$.

The grid points $Z_{k}^{(1)}$ are mapped into the function space of $K_{n}(Z)$; the closest location of the zero is sought according to the required condition that $\left|K_{n}(Z)\right|$ be a minimum. Denote this point by $Z_{i}$, and let the mesh within which the zero is located be defined by the points $\left(a_{i}, b_{i}\right),\left(a_{i}, b_{i+1}\right),\left(a_{i+1}, b_{i}\right),\left(a_{i+1}, b_{i+1}\right)$ [Fig. 1]. A more accurate location of the zero $(\bar{a}, \bar{b})$ within the mesh may now be sought by means of a double linear interpolation scheme. Letting

$$
K_{n}\left(a_{r}+i b_{s}\right)=\alpha_{r, s}+i \beta_{r, s},
$$

the approximate location of $(\bar{a}, \bar{b})$ lying within the mesh is then given by

$$
\begin{aligned}
& \bar{a}=a_{i}+c_{1}\left(a_{i+1}-a_{i}\right), \\
& \bar{b}=b_{i}+c_{2}\left(b_{j+1}-b_{j}\right),
\end{aligned}
$$

where

$$
\begin{gathered}
c_{1}=\frac{\alpha_{i, j}\left(\alpha_{i, j}-\alpha_{i+1, j}\right)+\beta_{i, j}\left(\beta_{i, j}-\beta_{i+1, i}\right)}{\left(\alpha_{i+1, j}-\alpha_{i, j}\right)^{2}+\left(\beta_{i+1, j}-\beta_{i, j}\right)^{2}}, \\
c_{2}=\frac{\alpha_{j, j}\left(\alpha_{i, j}-\alpha_{i, j+1}\right)+\beta_{i, j}\left(\beta_{i, j}-\beta_{j, j+1}\right)}{\left(\alpha_{i, j+1}-\alpha_{i, j}\right)^{2}+\left(\beta_{i, j+1}-\beta_{i, j}\right)^{2}} .
\end{gathered}
$$

Once the location of the zero, $Z_{0}^{(1)}=\bar{a}+i \bar{b}$ within the mesh is found, a second grid system with spacing $\Delta^{(2)}=\Delta^{(1)} / 10$ is established in the neighborhood of this point; upon repetition of the above calculations, a more accurate value $Z_{0}^{(2)}$ is obtained. 


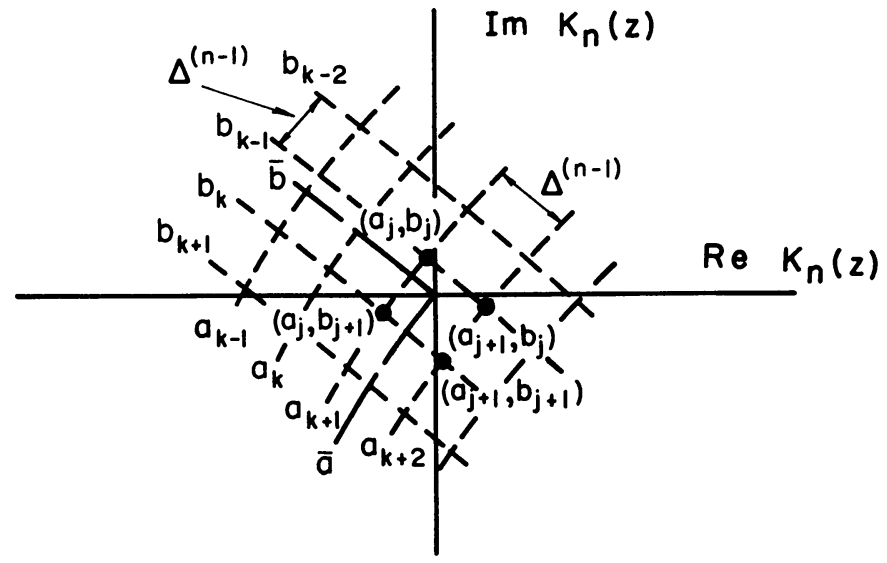

Figure 1

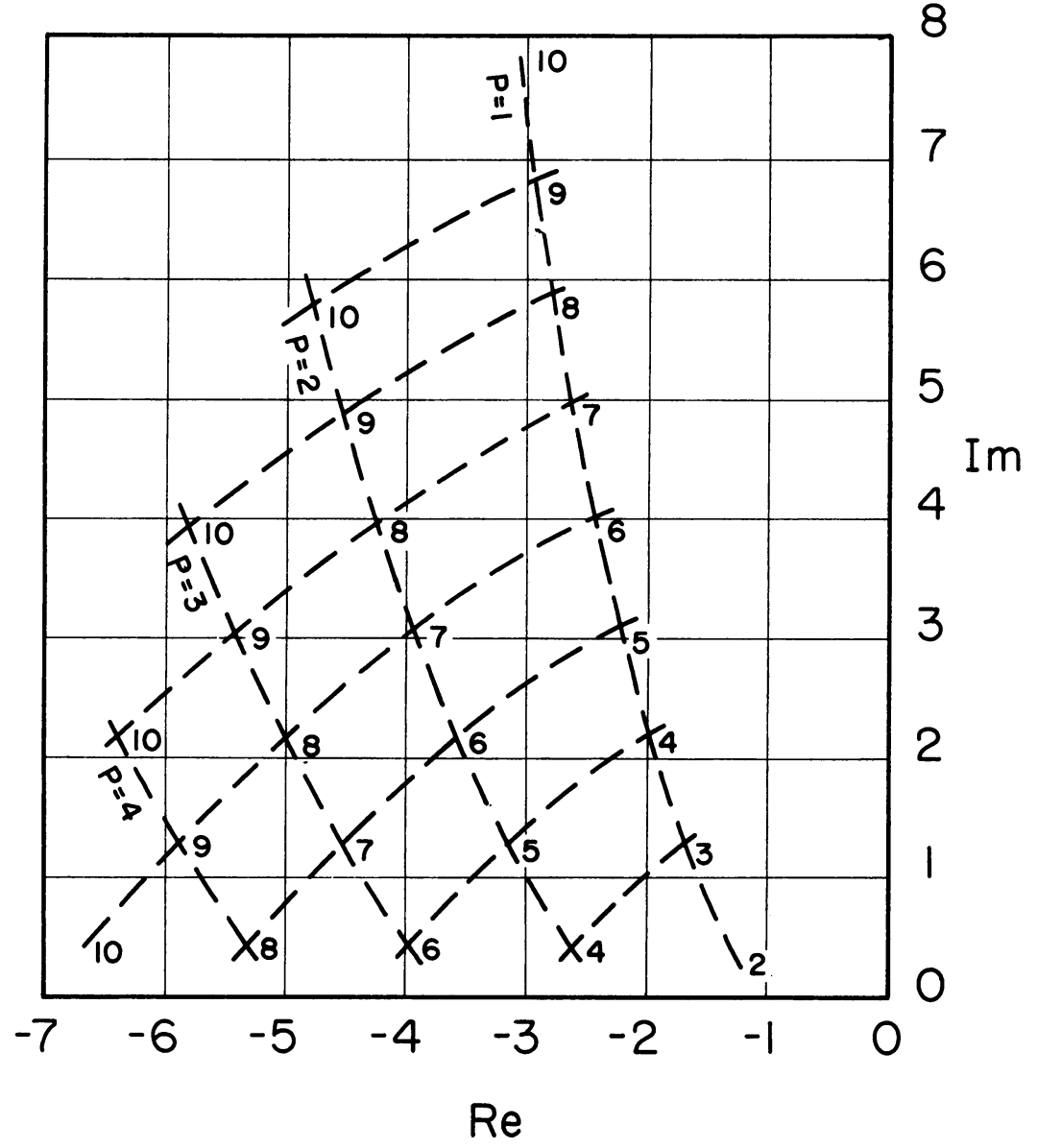

Figure 2 
TABLE I

\begin{tabular}{|c|c|c|c|c|}
\hline$n$ & $p$ & & $Z_{0}$ & \\
\hline 2 & 1,2 & -1.28137 & $3798 \pm 0.42948$ & $4965 i$ \\
\hline 3 & 1,2 & -1.68178 & $8805 \pm 1.30801$ & $2032 i$ \\
\hline 4 & $\begin{array}{l}1,3 \\
2,4\end{array}$ & $\begin{array}{l}-1.97816 \\
-2.62867\end{array}$ & $\begin{array}{l}1863 \pm 2.20437 \\
1168 \pm 0.43269\end{array}$ & $\begin{array}{l}1982 i \\
6649 i\end{array}$ \\
\hline 5 & $\begin{array}{l}1,3 \\
2,4\end{array}$ & $\begin{array}{l}-2.21862 \\
-3.13513\end{array}$ & $\begin{array}{l}6275 \pm 3.11308 \\
2845 \pm 1.30388\end{array}$ & $\begin{array}{l}2945 i \\
2398 i\end{array}$ \\
\hline 6 & $\begin{array}{l}1,4 \\
2,5 \\
3,6\end{array}$ & $\begin{array}{l}-2.42340 \\
-3.55109 \\
-3.96155\end{array}$ & $\begin{array}{l}4388 \pm 4.03096 \\
7904 \pm 2.18349 \\
8070 \pm 0.43334\end{array}$ & $\begin{array}{l}1581 i \\
5178 i \\
5409 i\end{array}$ \\
\hline 7 & $\begin{array}{l}1,4 \\
2,5 \\
3,6\end{array}$ & $\begin{array}{l}-2.60312 \\
-3.90812 \\
-4.51262\end{array}$ & $\begin{array}{l}6266 \pm 4.95596 \\
5740 \pm 3.07087 \\
6778 \pm 1.30277\end{array}$ & $\begin{array}{l}9607 i \\
1770 i \\
8842 i\end{array}$ \\
\hline 8 & $\begin{array}{l}1,5 \\
2,6 \\
3,7 \\
4,8\end{array}$ & $\begin{array}{l}-2.76414 \\
-4.22315 \\
-4.98827 \\
-5.29076\end{array}$ & $\begin{array}{l}2977 \pm 5.88671 \\
2279 \pm 3.96506 \\
8792 \pm 2.17708 \\
1293 \pm 0.43357\end{array}$ & $\begin{array}{l}2882 i \\
5969 i \\
2746 i \\
7695 i\end{array}$ \\
\hline 9 & $\begin{array}{l}1,5 \\
2,6 \\
3,7 \\
4,8\end{array}$ & $\begin{array}{l}-2.91058 \\
-4.50646 \\
-5.40974 \\
-5.86655\end{array}$ & $\begin{array}{l}2423 \pm 6.82219 \\
5946 \pm 4.86520 \\
7448 \pm 3.05654 \\
1467 \pm 1.30232\end{array}$ & $\begin{array}{l}0331 i \\
7143 i \\
4239 i \\
8327 i\end{array}$ \\
\hline 10 & $\begin{array}{l}1,6 \\
2,7 \\
3,8 \\
4,9 \\
5,10\end{array}$ & $\begin{array}{l}-3.04529 \\
-4.76484 \\
-5.79002 \\
-6.37839 \\
-6.61848\end{array}$ & $\begin{array}{l}3499 \pm 7.76165 \\
5373 \pm 5.77055 \\
7164 \pm 3.94097 \\
4971 \pm 2.17424 \\
1885 \pm 0.43368\end{array}$ & $\begin{array}{l}5671 i \\
5599 i \\
2616 i \\
8586 i \\
6206 i\end{array}$ \\
\hline
\end{tabular}

The procedure is then repeated successively giving a sequence of zeros, $\left\{Z_{0}^{(1)}\right.$, $\left.Z_{0}^{(2)}, \cdots, Z_{0}^{(n-1)}, Z_{0}^{(n)}, \cdots\right\}$. Each refinement of the grid, $\Delta^{(n)}=\Delta^{(n-1)} / 10$, yields a further significant figure in the location of the zero. The procedure may be repeated provided the number of significant figures is within an accuracy consistent with the accuracy of $K_{n}(Z)$.

3. Results. The zeros of $K_{n}(Z)$ were determined for integer orders $n=0(1) 10$. Calculations were performed on an IBM 360/50 computer using double precision which would indicate a possible accuracy of 15-17 significant figures. The procedure described above was repeated to yield complex zeros to 9D significant figures.

An estimate of the accuracy of the calculated zeros $Z=Z_{0}$ with respect to the true zeros $Z=Z_{0}^{*}$ may be obtained from the relation 


$$
K_{n}\left(Z_{0}\right) \approx K_{n}\left(Z_{0}^{*}\right)+K_{n}^{\prime}\left(Z_{0}^{*}\right) \cdot\left(Z_{0}-Z_{0}^{*}\right) .
$$

The order of the error $\left|Z_{0}-Z_{0}^{*}\right|$ is then determined from

$$
\left|Z_{0}-Z_{0}^{*}\right| \approx\left|K_{n}\left(Z_{0}\right)\right| /\left|K_{n}^{\prime}\left(Z_{0}^{*}\right)\right| \approx\left|K_{n}\left(Z_{0}\right)\right| /\left|K_{n}^{\prime}\left(Z_{0}\right)\right| \text {. }
$$

For all the values $Z=Z_{0}$ presented, the remainders are $\left|K_{n}\left(Z_{0}\right)\right|<O\left(10^{-10}\right)$, while all $\left|K_{n}^{\prime}\left(Z_{0}\right)\right|$ are found to be of order unity; hence, $\left|Z_{0}-Z_{0}^{*}\right| \approx O\left(10^{-10}\right)<$ $O\left(10^{-9}\right)$, thereby indicating an accuracy of at least 9D significant figures.

It is noted that $K_{n}$ has no finite zeros for $n=0,1$. Furthermore, the number $N$ of zeros for each integer $n$, in the pair of quadrants $\operatorname{Re} Z<0$, agrees with the number given by Watson [6, p. $511 \mathrm{ff}$.], viz. "... the number is the even integer which is nearest to $n-\frac{1}{2}$ "'. Thus, denoting the zeros as $Z_{p}$, for each $n$,

$$
p=1,2,3, \cdots, N
$$

where

$$
\begin{array}{rlrl}
N & =n, & & n \text { even, } \\
& =n-1, \quad n \text { odd. }
\end{array}
$$

The complex zeros of $K_{n}(Z)$ are given in Table I. For $n \leqq 5$, upon interchanging the real and imaginary parts, it is observed that the tabulated zeros are in agreement to all significant figures with the values of the zeros of Hankel functions $H_{n}^{(1)}(Z)$ given by Döring [5] in the cases where the zeros coincide.

It is also apparent that the zeros of $K_{n}(Z)$ fall into a definite pattern as is shown, for the positive quadrant, in Fig. 2, where the zeros are observed to lie at the intersections of the suggestive curves.

Department of Civil Engineering

The City College of CUNY

139th Street and Convent Avenue

New York, New York 10031

1. F. W. J. Olver, "The asymptotic expansion of Bessel functions of large order," Philos. Trans. Roy. Soc. London Ser. A, v. 247, 1954, pp. 328-368. MR 16, 696.

2. Y. L. Luke, The Special Functions and Their Approximations. Vol. I, Math. in Sci. and Engineering, vol. 53, Academic Press, New York, 1969. MR 39 \#3039.

3. J. L. FIELDS, "A linear scheme for rational approximations," J. Approximation Theory (To appear.)

4. J. A. Cochran \& J. N. Hoffspiegel, "Numerical techniques for finding $\nu$-zeros of Hankel functions," Math. Comp., v. 24, 1970, pp. 413-422. MR 42 \#038.

5. B. Döring, "Complex zeros of cylinder functions," Math. Comp., v. 20, 1966, pp. 215-222. MR 33 \#857.

6. G. N. Watson, A Treatise on the Theory of Bessel Functions, Cambridge Univ. Press, Cambridge, 1944. MR 6, 64. 1949.

7. E. Goursat, Cours d'Analyse Mathématique. Vol. 2, 7th ed., Gauthier-Villars, Paris, 